\title{
Discrete-Time Queueing Model of Age of Information With Multiple Information Sources
}

\author{
Nail Akar ${ }^{\circledR}$, Member, IEEE, and Ozancan Dogan ${ }^{(1)}$
}

\begin{abstract}
Information freshness in IoT-based status update systems has recently been studied through the Age of Information (AoI) and Peak AoI (PAoI) performance metrics. In this article, we study a discrete-time server arising in multisource IoT systems, which accepts incoming information packets from multiple information sources so as to be forwarded to a remote monitor for status update purposes. Under the assumption of Bernoulli information packet arrivals and a common general discrete phase-type service time distribution across all the sources, we numerically obtain the exact per-source distributions of AoI and PAoI in matrix-geometric form for three different queueing disciplines: 1) nonpreemptive bufferless; 2) preemptive bufferless; and 3) nonpreemptive single buffer with replacement. The proposed numerical algorithm employs the theory of discretetime Markov chains of quasi-birth-death type and is matrix analytical. Numerical examples are provided to validate the accuracy and effectiveness of the proposed queueing model. We also present a numerical example on the optimum choice of the Bernoulli parameters in a practical IoT system with two sources with diverse AoI requirements.
\end{abstract}

Index Terms-Age of Information (AoI), discrete-time queues, Peak AoI (PAoI), Markov chains of quasi-birth-death (QBD) type.

\section{INTRODUCTION}

$\mathbf{I}^{\mathrm{N}}$ $\mathrm{N}$ A NETWORKED control and monitoring IoT-based system, it is of utmost importance to deliver timely status updates and thus keep the information fresh, for stable operation. Performance metrics using the Age of Information (AoI) and Peak AoI (PAoI) processes have first been proposed in [1]-[3] in continuous time, for quantitative assessment of information freshness in status update systems. Since then, there has been a surge of interest in AoI research in terms of development of queueing models [4]-[9] and AoI optimization [10]-[14]. In the discrete-time setting, for each information source, there is an underlying stochastic process that is randomly sampled, and the sampled values are transmitted in the form of information packets toward a remote monitor via a packet-based communications network. Subsequently, for each information source, there is an individual stochastic process, called the AoI process (or sequence), which is maintained at the monitor that keeps track of the time elapsed

Manuscript received July 21, 2020; revised November 24, 2020; accepted January 9, 2021. Date of publication January 22, 2021; date of current version September 23, 2021. The work of Ozancan Dogan was supported by the $5 \mathrm{G}$ and Beyond scholarship granted by the Information and Communication Technologies of Turkey and Vodafone Turkey. (Corresponding author: Nail Akar.)

The authors are with the Electrical and Electronics Engineering Department, Bilkent University, 06800 Ankara, Turkey (e-mail: akar@ee.bilkent.edu.tr).

Digital Object Identifier 10.1109/JIOT.2021.3053768

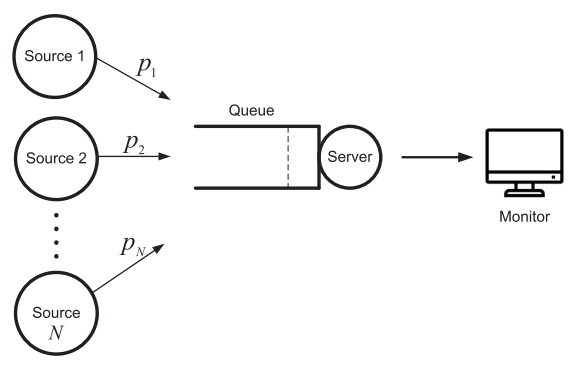

Fig. 1. $\quad N$ information sources sending status update messages through a server toward a remote monitor.

since the generation of the last successfully received update packet. This per-source AoI process is a cyclic process that increases in time with unit steps until the end of a cycle at which a packet reception occurs and subsequently the AoI process experiences an abrupt downward jump. On the other hand, the PAoI process is obtained by sampling the AoI sequence at the embedded epochs just before the downward jumps during each cycle of the AoI process [15].

Slotted discrete-time operation in industrial IoT infrastructures has been quite commonplace. Scalability and reliability of the infrastructure required for most industrial IoT use cases can be enhanced, thanks to collision friendliness of the timeslotted operation. For example, the IETF 6TiSCH provides link layer interfaces that support a time-slotted MAC layer with reduced collisions and is thus well suited for deterministic industrial applications [16]. As another recent example, time-slotted LoRaWAN has recently been proposed in [17] for the LoRa-based low-power wide-area network scenario. Analytical modeling of AoI and PAoI in IoT systems using time-slotted operation is, therefore, crucial. Although distributional results exist in the literature for continuous-time operation [9], results for discrete-time slotted operation are less mature except for the ones that provide average AoI and PAoI values in relatively limited settings [18]. The focus of this article is on a time-slotted IoT-based information update system given in Fig. 1 comprising $N$ independent information sources each equipped with a sensor, a queue-server combination local to the sources, and a remote monitor. In this framework, source- $n, n=1, \ldots, N$, generates information packets in discrete time according to a Bernoulli process with parameter $p_{n}$ containing sensed data and a timestamp. The server is in charge of sending the information packets to the monitor via a communications network that introduces random 
delays, i.e., service time of packets, and the monitor immediately sends back positive acknowledgments to the server. As an example (also see [8]), consider a vehicle consisting of various sensors each of which is to generate status update messages to a gateway located on the vehicle that is in charge of relaying these messages wirelessly to a destination whether it be another vehicle or a networked control or monitoring system away from the vehicle. The status update messages are continuously generated and queued at the gateway and it is crucial to use effective queue management at the gateway so as to optimize performance metrics involving per-sensor AoI or PAoI values.

We assume in this article that the service times of source packets, denoted by $\Theta$, are distributed according to a common discrete phase-type (DPH) distribution [19]. Generality of DPH distributions makes it possible to use the proposed AoI model in a broad range of scenarios. Particularly, we study the following three queueing disciplines employed at the server.

1) Nonpreemptive bufferless (NPB) system for which one of the generated information packets at each time instant is selected uniformly at random, or randomly in short, to be placed in service when the server is idle. Otherwise, all packets are discarded.

2) Preemptive bufferless (PB) system in which one of the generated information packets at each time instant (selected randomly) is always placed in service while possibly preempting the one in service.

3) Nonpreemptive single buffer with replacement (NPSBR) system for which we have a buffer holding one information packet only in the waiting room and one of the generated information packets at each time instant is randomly selected to replace the one in the waiting room. If there are no packets in the buffer, this information packet is placed in service if the server is idle or otherwise, it joins the waiting room.

FIFO-based queue management with large buffers for which one might have multiple packets in the waiting room from the same source is known to work poorly in terms of AoI [20]. However, keeping the most recent packet for each source has been shown to be advantageous in terms of AoI [4]. Such systems with per-source buffering are not only harder to analyze but also a need arises for scheduling among the waiting packets when the server is to decide which waiting packet to transmit. Modeling of queues with per-source buffering and scheduling is deliberately left for future work. For the vehicle example employing one of the three queueing schemes mentioned above, the gateway is assumed to have either no buffering or it holds the most recent status update irrespective of the source. In this way, a simple-toimplement source-agnostic queueing scheme is envisioned for the gateway.

As the main contribution of this article, we propose a novel analytical modeling technique based on the theory of quasibirth-death (QBD) processes, or QBDs in short, to derive the exact distributions of per-source AoI and PAoI in discrete time, for the bufferless NPB and PB systems as well as the single buffer NPSBR system with quite general DPHdistributed service times. Moreover, the proposed method is matrix analytical, i.e., it is based on numerically stable vectormatrix operations, and is well suited for use in time-slotted communication systems involving the modeling of AoI.

The remainder of this article is organized as follows. In Section II, related work is presented. Section III addresses DPH distributions, and QBDs along with their steady-state solutions. Section IV presents the proposed queueing model for the bufferless queueing disciplines NPB and PB as well as the single-buffer system NPSBR. Numerical examples are provided to validate the accuracy and effectiveness of the proposed queueing model in Section V. Finally, we conclude in Section VI.

\section{RElated WORK ON QUEUEING MOdELS FOR AOI AND PAOI}

The AoI concept was first introduced in [2] as a singlesource, single-server $M / M / 1$ queueing model. The case of multiple sources is then investigated in [21] in the same setting. Variations of this single-server queueing model in Fig. 1 for AoI has caught the attention of researchers and practitioners for modeling IoT-based status update systems in the literature [2], [20]. The majority of the existing queueing models on AoI or PAoI are for continuous-time operation.

Let us first briefly overview the literature on single-source continuous-time models. In [2], the mean AoI is obtained for the single-source $M / M / 1, M / D / 1$, and $D / M / 1$ queues with infinite buffer capacity and FCFS scheduling. Although current packet-switched communication networks, such as the Internet, employ large buffers and FCFS scheduling at the routers, their use is shown to have adverse effects on AoI performance figures in moderate to high load regimes [2]. The distributions of AoI and PAoI are, therefore, studied in [6] for the case of small buffers, including the conventional $M / M / 1 / 1$ and $M / M / 1 / 2$ queues, as well as the nonpreemptive LCFS $M / M / 1 / 2^{*}$ queue. Exact expressions for the stationary distributions of AoI and PAoI for a very wide class of single-source information update systems are given in [9]. Akar et al. [22] obtained the exact distributions of AoI and PAoI in a bufferless $P H / P H / 1 / 1$ system with probabilistic preemption as well as a single-buffer $M / P H / 1 / 2$ system that allows probabilistic replacement of the waiting packet by a newer packet arrival.

Next, we give an overview of the existing literature on multisource status update systems in continuous time. Mean PAoI expressions for $M / G / 1$ and $M / G / 1 / 1$ systems with heterogeneous service time requirements are presented in [11]. Yates and Kaul [8] investigated the multisource $M / M / 1$ model with FCFS as well as two disciplines: 1) PB and 2) NPSBR using the theory of stochastic hybrid systems (SHS) and obtained exact expressions for the mean AoI. A preemptive $M / G / 1 / 1$ queue is considered in [23] with a common service time for all sources in which expressions for the mean AoI and PAoI are derived. Farazi et al. [24] allowed self-preemption in which case mean AoI expressions are derived for each source using SHS-based techniques whereas Moltafet et al. [25] considered a two-source $M / M / 1 / 2$ queueing system in which a packet waiting in the queue can be replaced only by a newly arriving packet from the same source, again using SHS. A 
nonpreemptive $M / M / 1 / m$ with common service times across sources is again studied by SHS in [26] and mean AoI expressions are derived. A more general hyperexponential service time distribution for each class is considered in [27] for an $M / H_{2} / 1 / 1$ NPB queue to obtain an expression for the mean AoI per class.

The existing research on discrete-time AoI queueing models is less mature in comparison with continuous-time models. A discrete-time queueing model with Bernoulli arrivals and geometric service times, using FCFS and nonpreemptive LCFS scheduling, is presented in [28] with expressions for the mean AoI and PAoI values. Tripathi et al. [18] studied the FCFS-type $\mathrm{Ber} / G / 1$ queue and derived explicit expressions for average AoI and PAoI and also mean AoI expressions for the discrete-time LCFS queue. For the single-source case, Kosta et al. [29] considered the FCFS Geom/Geom/1 queue and obtained closed-form expressions of the generating functions and the stationary distributions of the AoI and PAoI and provided a methodology for analyzing more general nonlinear age functions.

\section{Discrete Phase Type Distributions AND DTMCS OF QUASI-BIRTH-DEATH TYPE}

We first present the notations. Uppercase bold letters are used to denote real-valued matrices. Lowercase bold (plain) letters or symbols are used to denote real-valued vectors (scalars). The $(i, j)$ th element of a matrix $\boldsymbol{A}$ is denoted by $A(i, j)$. Similarly, the $i$ th element of a row or column vector $\boldsymbol{a}$ is denoted by $a(i)$. The notations $\mathbf{0}_{k \times \ell}, \boldsymbol{I}_{m}$, and $\mathbf{1}_{n}$ denote a matrix of zeros of size $k \times l$, an identity matrix of size $m$, and a column matrix of ones of size $n$, respectively. When used without a subscript, we leave it to the reader to infer the size information, from the context. The function $u_{k}$ stands for the discrete-time unit step function, i.e., $u_{k}=1, k=0,1, \ldots$ and is zero otherwise. The function $\delta_{k}$ stands for the discretetime unit impulse function, i.e., $\delta_{k}=1$ for $k=0$ and is zero, otherwise.

The DPH distribution (see [19], [30], and [31]) is obtained as the time until absorption (denoted by $X$ ) in a discrete-state discrete-time Markov chain (DTMC) with $r$ transient states numbered as $1,2, \ldots, r$, one absorbing state $r+1$, its initial probability vector denoted by $\boldsymbol{\alpha}$, and its probability transition matrix given as

$$
\left[\begin{array}{ll}
\boldsymbol{T} & \boldsymbol{t} \\
0 & 1
\end{array}\right]
$$

for an $r \times r$ substochastic matrix $\boldsymbol{T}$ and an $r \times 1$ vector $\boldsymbol{t}=(\boldsymbol{I}-$ T)1. In this case, $X \sim \operatorname{DPH}(\boldsymbol{\alpha}, \boldsymbol{T}, \boldsymbol{t})$, which has a probability mass function (pmf) $p_{X}(\ell)$ of the form

$$
p_{X}(\ell)=\operatorname{Pr}\{X=\ell\}=\alpha \boldsymbol{T}^{\ell-1} \boldsymbol{t}_{\ell-1} .
$$

A discrete positive random variable $X$ with finite support pmf, i.e., $p_{X}(\ell)=0$ when $\ell>r$, and $G_{X}(\ell)=\sum_{i=\ell}^{r} p_{X}(i)$, is of DPH-type of order $r$, i.e., $X \sim \operatorname{DPH}(\boldsymbol{\alpha}, \boldsymbol{T}, \boldsymbol{t})$ where

$$
\boldsymbol{\alpha}=\left[\begin{array}{llll}
1 & 0 & \cdots & 0
\end{array}\right]^{T}
$$

$$
\boldsymbol{T}=\left[\begin{array}{ccccc}
0 & T(1,2) & 0 & \ldots & 0 \\
0 & 0 & T(2,3) & \cdots & 0 \\
\vdots & \vdots & \ddots & \ddots & 0 \\
0 & 0 & \cdots & 0 & T(r-1, r) \\
0 & 0 & \cdots & 0 & 0
\end{array}\right]
$$

where $T(\ell, \ell+1)=1-\left(p_{X}(\ell) / G_{X}(\ell)\right), 1 \leq \ell \leq r-1$, and $\boldsymbol{t}=(\boldsymbol{I}-\boldsymbol{T}) \mathbf{1}$. In this article, for the sake of generality, service time of packets is assumed to be of DPH type that includes the deterministic distribution, uniform distribution, geometric distribution, and mixed geometric distribution, as its subcases.

A discrete random variable $X$ is said to possess a matrix geometric (MG) distribution of order $r$, i.e., $X \sim M G(\boldsymbol{c}, \boldsymbol{A}, \boldsymbol{b}, d)$, if its pmf, denoted by $p_{X}(\ell)$, is of the form [31]

$$
p_{X}(\ell)=\boldsymbol{c} \boldsymbol{A}^{\ell-1} \boldsymbol{b} u_{\ell-1}+d \delta_{\ell}
$$

for a square matrix $A$ of size $r$, which is not necessarily a substochastic matrix as in DPH. DPH distributions are subcases of MG distributions but the converse may not be true. The probability generating function (pgf) of $X$, denoted by $p_{X}^{*}(z)$, is then of the form

$$
p_{X}^{*}(z)=\sum_{\ell=0}^{\infty} p_{X}(\ell) z^{\ell}=\boldsymbol{c}\left(z^{-1} \boldsymbol{I}-\boldsymbol{A}\right)^{-1} \boldsymbol{b}+d .
$$

The factorial moments of MG distributions can be found by differentiating (5) successively with respect to $z$ and substituting $z=1$. Consequently, the $i$ th factorial moment of an MG-distributed random variable $X$ can be found through the following expression (see [31]):

$$
E[X(X-1) \cdots(X-i+1)]=i ! c(I-\boldsymbol{A})^{-i-1} \boldsymbol{A}^{i-1} \boldsymbol{b} .
$$

An infinite QBD-type DTMC

$$
X_{k}=\left(L_{k}, P_{k}\right) \sim \operatorname{QBD}\left(\boldsymbol{B}_{\mathbf{0}}, \boldsymbol{B}_{\mathbf{1}}, \boldsymbol{A}_{\mathbf{0}}, \boldsymbol{A}_{\mathbf{1}}, \boldsymbol{A}_{\mathbf{2}}\right), k \geq 0
$$

is a 2-D chain with $\left(L_{k}, P_{k}\right) \in\{(i, j): 0 \leq i<\infty, 1 \leq j \leq m\}$, where $L_{k}$ represents the level sequence of the QBD, $P_{k}$ stands for the phase sequence, and $X_{k}$ has an irreducible probability transition matrix $\boldsymbol{P}$ of the following canonical block tridiagonal form:

$$
\boldsymbol{P}=\left[\begin{array}{cccc}
\boldsymbol{B}_{0} & A_{0} & & \\
B_{1} & A_{1} & A_{0} & \\
& A_{2} & A_{1} & \ddots \\
& & A_{2} & \ddots \\
& & & \ddots
\end{array}\right]
$$

with $\boldsymbol{B}_{\mathbf{0}}, \boldsymbol{B}_{\mathbf{1}}, \boldsymbol{A}_{\mathbf{0}}, \boldsymbol{A}_{\mathbf{1}}$, and $\boldsymbol{A}_{\mathbf{2}}$ being $m \times m$ nonnegative matrices. Finite QBD chains where the level of the chain stays within a bound is outside the scope of this article. The stationary probability vector $\pi=\left[\boldsymbol{\pi}_{0}, \boldsymbol{\pi}_{1}, \ldots\right]$ where $\pi_{k}$, of size $1 \times m$, is the solution vector for level $k$, is the unique solution to the following equations:

$$
\boldsymbol{\pi}=\boldsymbol{\pi} \boldsymbol{P}, \sum_{k=0}^{\infty} \pi_{k} \mathbf{1}=1 .
$$


The stationary solution (when it exists) has a matrix-geometric form [32], i.e.,

$$
\pi_{k}=\pi_{0} \boldsymbol{R}^{k} u_{k}
$$

where the matrix-geometric rate matrix $\boldsymbol{R}$ (all its eigenvalues being inside the unit circle) is the unique minimal nonnegative solution of the following quadratic matrix equation:

$$
R=A_{0}+R A_{1}+R^{2} A_{2} \text {. }
$$

Once $\boldsymbol{R}$ is computed, the boundary vector $\boldsymbol{\pi}_{\mathbf{0}}$ in (10) can be found from the solution of the following linear matrix equations [32]:

$$
\boldsymbol{\pi}_{\mathbf{0}}=\boldsymbol{\pi}_{\mathbf{0}}\left(\boldsymbol{B}_{\mathbf{0}}+\boldsymbol{R} \boldsymbol{B}_{1}\right), \boldsymbol{\pi}_{\mathbf{0}}(\boldsymbol{I}-\boldsymbol{R})^{-1} \mathbf{1}=1 .
$$

Several algorithms with computational complexity $\mathcal{O}\left(\mathrm{m}^{3}\right)$ are known in the literature for obtaining the rate matrix $\boldsymbol{R}$, including the logarithmic reduction procedure [33], or the invariant subspace algorithm using the ordered Schur decomposition [34], both algorithms being computationally efficient and stable. In the current paper's numerical examples, we use the latter algorithm.

Let $L$ be the random variable associated with the level sequence $L_{k}$ of the QBD process $X_{k}$ in the steady state with the following expression for its $\operatorname{pmf} p_{L}(\ell)$ :

$$
p_{L}(\ell)=\lim _{k \rightarrow \infty} \operatorname{Pr}\left\{L_{k}=\ell\right\}=\boldsymbol{\pi}_{\mathbf{0}} \boldsymbol{R}^{\ell} \mathbf{1} u_{\ell}
$$

Therefore, $L \sim \operatorname{MG}\left(\pi_{0} R, R, 1, \pi_{0} \mathbf{1}\right)$. Another random variable to be needed in AoI calculations is the level sequence in the steady state but restricted to a particular subset of the set of phases. For this purpose, we define $L^{\mathcal{S}}$ to be the steady-state random variable associated with the level sequence $L_{k}$ of the QBD restricted to a particular subset $\mathcal{S} \subset\{1,2, \ldots, m\}$. In this case

$$
p_{L} \mathcal{S}(\ell)=\lim _{k \rightarrow \infty} \operatorname{Pr}\left\{L_{k}=\ell \mid P_{k} \in \mathcal{S}\right\} .
$$

It is not difficult to show that

$$
L^{\mathcal{S}} \sim \operatorname{MG}\left(\kappa \pi_{0} \boldsymbol{R}, \boldsymbol{R}, \boldsymbol{h}, \kappa \pi_{0} \boldsymbol{h}\right)
$$

where $\boldsymbol{h}_{i}=1$ if $i \in \mathcal{S}$ and is zero otherwise, and

$$
\kappa=\left(\pi_{0}(\boldsymbol{I}-\boldsymbol{R})^{-1} \boldsymbol{h}\right)^{-1}
$$

One final random variable in the QBD context to be needed in PAoI calculations is the level sequence in the steady state but restricted to the pretransition epochs from a particular subset of the set of phases to a certain phase selected outside this subset. To this end, we define $L^{\mathcal{S}, j}$ to be the steady-state random variable associated with the level sequence $L_{k}$ of the QBD process $X_{k}$ restricted to the pretransition epochs to a certain phase $j$ from a designated subset $\mathcal{S} \subset\{1,2, \ldots, m\}$ with $j \notin \mathcal{S}$ assuming such transitions are possible. In this case

$$
\begin{aligned}
& p_{L} \mathcal{S}, j(\ell)=\lim _{k \rightarrow \infty} \operatorname{Pr}\left\{L_{k}=\ell \mid P_{k} \in \mathcal{S}, P_{k+1}=j\right\} \\
& =\frac{\lim _{k \rightarrow \infty} \operatorname{Pr}\left\{L_{k}=\ell, P_{k} \in \mathcal{S}, P_{k+1}=j\right\}}{\lim _{k \rightarrow \infty} \operatorname{Pr}\left\{P_{k} \in \mathcal{S}, P_{k+1}=j\right\}} \\
& =\frac{\sum_{i \in \mathcal{S}} \lim _{k \rightarrow \infty} \operatorname{Pr}\left\{L_{k}=\ell, P_{k}=i, P_{k+1}=j\right\}}{\sum_{i \in \mathcal{S}} \lim _{k \rightarrow \infty} \operatorname{Pr}\left\{P_{k}=i, P_{k+1}=j\right\}}
\end{aligned}
$$

$$
=\frac{\sum_{i \in \mathcal{S}} \pi_{\ell}(i) \operatorname{Pr}\left\{P_{k+1}=j \mid P_{k}=i, L_{k}=\ell\right\}}{\sum_{i \in \mathcal{S}} \operatorname{Pr}\left\{P_{k+1}=j \mid P_{k}=i\right\}} .
$$

Assuming that these transitions to the set $j$ are possible only when the level is being incremented (which will be shown to be the case for PAoI calculations), one can show that

$$
p_{L} \mathcal{S}(\ell)=\frac{\sum_{i \in \mathcal{S}} \pi_{\ell}(i) A_{0}(i, j)}{\sum_{\ell=1}^{\infty} \sum_{i \in \mathcal{S}} \pi_{\ell}(i) A_{0}(i, j)} .
$$

Therefore, in this case

$$
L^{\mathcal{S}, j} \sim \operatorname{MG}\left(\kappa \pi_{0} \boldsymbol{R}, \boldsymbol{R}, \boldsymbol{h}, \kappa \pi_{0} \boldsymbol{h}\right)
$$

where $\boldsymbol{h}_{i}=A_{0}(i, j)$ if $i \in \mathcal{S}$ and is zero otherwise, and $\kappa$ is defined as in (16). Therefore, factorial moments (hence also ordinary moments) of $L^{\mathcal{S}}$ and $L^{\mathcal{S}, j}$ can easily be computed from the expression (6) for any subset $\mathcal{S}$ and any phase $j$.

\section{Discrete-Time Queueing Modeling OF AOI AND PAOI}

We first describe the AoI and PAoI processes (sequences) for the three queueing disciplines NPB, PB, and NPSBR, accompanied by an illustrative example. For this purpose, a successful information packet is first defined as one, which is received successfully by the monitor; others those that could not start service or that were preempted while in service (this latter situation being specific to $\mathrm{PB}$ ) are called unsuccessful packets. Let $t_{j}^{(n)}$ denote the arrival instant of the $j$ th, $j \geq 1$ successful source- $n$ information packet arriving at the server and let $\delta_{j}^{(n)}, j \geq 1$ denote the reception instant at the monitor of the $j$ th successful source- $n$ information packet. We denote by $\Delta_{k}^{(n)}, k=0,1, \ldots$, the discrete-time discrete-valued random sequence representing the AoI for source- $n$ at discrete-time instant $k$ with a given initial condition $\Delta_{0}^{(n)}$.

1) When $k \geq 0, \Delta_{k}^{(n)}$ is incremented by one at each time instant until the first source- $n$ successful packet reception occurring at instant $k=\delta_{1}^{(n)}$ at which it is again first incremented to yield the PAoI value $\Phi_{1}^{(n)}$ where $\Phi_{j}^{(n)}$ denotes the PAoI process for source- $n$, which is a discrete-time discrete-valued random sequence associated with the AoI just before the reception of the $j$ th successful source- $n$ information packet.

2) Moreover, at instant $k=\delta_{1}^{(n)}$, just after the packet's reception, $\Delta_{k}^{(n)}$ is set to $D_{1}^{(n)}$ where $D_{j}^{(n)}$ is the time spent in the system (queue wait plus service) by the $j$ th successful source- $n$ information packet i.e., $D_{j}^{(n)}=$ $\delta_{j}^{(n)}-t_{j}^{(n)}, j \geq 1$.

3) Following this packet reception, $\Delta_{k}^{(n)}$ is again incremented by one at each time instant until the reception of the second source- $n$ successful packet and the AoI random sequence is obtained by repeating this pattern forever.

Discrete-time Markov modeling is quite different than that of continuous time since in the latter, only one event can happen at a given time. However, in discrete time, multiple events can happen at the same time instant. Therefore, the sequence of events happening at each time instant is crucial for modeling. Depending on the particular application or implementation, 
TABLE I

Assumed SEQuence of Events at Time Instant $k$

\begin{tabular}{|c|c|}
\hline Event & Description \\
\hline 1 & $\begin{array}{l}\text { At the beginning of instant } k \text {, the AoI values } \Delta_{k}^{(n)}, 1 \leq n \leq \\
N \text { are incremented. }\end{array}$ \\
\hline 2 & $\begin{array}{l}\text { The service time of the packet, say source- } n \text { in service, being } \\
\text { over or not is checked, if over then the server is placed into } \\
\text { the idle state and the AoI value } \Delta_{k}^{(n)} \text { is updated as } D_{j}^{(n)} \text { if } \\
\text { this packet turns out to be the } j^{\text {th }} \text { successful source- } n \text { packet. }\end{array}$ \\
\hline 3 & $\begin{array}{l}\text { - In NPB, if the server is idle, then one of the newcoming } \\
\text { packet arrivals is randomly chosen for starting service. } \\
\text { Otherwise, all packet arrivals are discarded. } \\
\text { - The situation is the same in PB when the server is idle. } \\
\text { Otherwise, one of the packet arrivals is randomly chosen } \\
\text { for preempting the packet in service. } \\
\text { - In NPSBR, one of the packet arrivals is always first } \\
\text { written into the waiting room (possibly replacing the one } \\
\text { in the waiting room). Subsequently, the packet in the } \\
\text { waiting room starts to receive service if the server is idle. } \\
\text { Otherwise, it will be held at the waiting room until the } \\
\text { next time instant. }\end{array}$ \\
\hline
\end{tabular}

different event sequences might arise. Although the methodology can be easily be extended to different event sequences, we focus on the particular sequence of events at time instant $k, k=0,1, \ldots$, which is described in Table I.

As stated above, in our model, we assume that only one of the newcoming arrivals can be picked and processed and it is not possible to place one of the newcoming arrivals in service and another one in the waiting room in the case of NPSBR at the same time instant. We leave such extensions for future research.

Table II provides the sample values of the random sequences $\Delta_{k}^{(n)}, k \geq 0$, for $n=1,2$ for a two-source example with zero initial conditions for AoI values for the three queueing disciplines NPB, PB, and NPSBR. For this particular example, we assume that source-1 packets arrive periodically at instants $k=1,5,9, \ldots$, with the first five packets indexed $1 a-1 e$ having service times 5, 2, 7, 3, and 1, respectively, and source-2 packets also arrive periodically at instants $k=1,7,13, \ldots$, with the first four packets $2 a-2 d$ having service times 4,4 , 2 , and 5 , respectively. We assume that the packet generated by source-1 (source-2) is to be chosen when the two sources generate packets simultaneously at $k=1(k=13)$. The following explanations are given for each of the three disciplines to follow the sample evolution of the AoI and PAOI sequences.

1) For NPB, at $k=1$, packet $l a$ is placed in service and $\Delta_{k}^{(1)}$ is initially incremented by one at each instant until the reception of $l a$ at $k=6$ leading to $\Phi_{1}^{(1)}=6$. In the meantime, packet $l b$ is discarded at $k=5$ since the server is busy upon its arrival. Subsequently, $\Delta_{k}^{(1)}$ is again incremented by one but rising from the value 5 (service time of $1 a$ ) until the reception of $l e$ at $k=18$. The packets $l b$ and $l c$ are discarded since they found the server busy and packet $l d$ is not picked against packet $2 c$. Similarly, packet $2 a$ is not picked against $l a$ and packet $2 b$ is placed in service at $k=4$, and therefore, $\Delta_{k}^{(2)}$ is initially incremented by one at each instant until the reception of $2 b$ at $k=11$ giving rise to $\Phi_{1}^{(2)}=11$. Subsequently, $\Delta_{k}^{(2)}$ is again incremented by one but rising from the value 4 (service time of $2 b$ ) until the reception of $2 c$ at $k=15$.

2) For $\mathrm{PB}$, at $k=1$, packet $l a$ is placed in service but is preempted by $l b$, which is received at $k=7$. Thus, $\Delta_{k}^{(1)}$ is incremented by one until $k=7$ leading to $\Phi_{1}^{(1)}=7$. Packet $2 b$ joins service at $k=7$ that is preempted by $1 c$, which is also eventually preempted by the successful packet $2 c$ received at $k=15$. Therefore, $\Delta_{k}^{(2)}$ is incremented by one at each instant until $k=15$ giving rise to $\Phi_{1}^{(2)}=15$. Following this, packet $1 e$ joins service at $k=17$ and is received at $k=18$. Thus, subsequently, $\Delta_{k}^{(1)}$ is again incremented by one but rising from the value 5 (service time of $1 a$ ) until the reception of $l e$ at $k=18$. Thus, $\Delta_{k}^{(1)}$ is incremented by one until $k=18$ leading to $\Phi_{2}^{(1)}=13$ while rising from the value of 2 at $k=7$.

3) For NPSBR, at $k=1$, packet $1 a$ is placed in service and is received at $k=6$ whereas $l b$ joins the waiting room at $k=5$ and starts receiving service at $k=6$, which gets to complete at $k=8$. Packet $2 b$ joins the waiting room at $k=7$ and starts to receive service at $k=8$. Thus, the first successful source-2 reception occurs at $k=12$ at which packet $l c$ starts receiving service, which gets to complete at $k=19$. Packet $2 c$ joins the waiting room at $k=13$ but is replaced with packet $l e$ that joins service at $k=17$ and subsequently completes at $k=18$.

We use the notation $\Delta^{(n)}, \Phi^{(n)}$, and $D^{(n)}$, to denote the steady-state random variables associated with the processes $\Delta_{k}^{(n)}, \Phi_{j}^{(n)}$, and $D_{j}^{(n)}$, respectively. For ease of notation, we tag a single source out of all sources, say source-1, and drop the superscript while writing the steady-state pmf for the random variables $\Delta=\Delta^{(1)}$ and $\Phi=\Phi^{(1)}$, respectively

$$
\begin{array}{ll}
p_{\Delta}(\ell)=\lim _{k \rightarrow \infty} \operatorname{Pr}\left\{\Delta_{k}^{(1)}=\ell\right\}, & \ell \geq 0 \\
p_{\Phi}(\ell)=\lim _{j \rightarrow \infty} \operatorname{Pr}\left\{\Phi_{j}^{(1)}=\ell\right\}, & \ell \geq 0 .
\end{array}
$$

We also denote by $F_{\Delta^{(n)}}(\ell)$ and $F_{\Phi^{(n)}}(\ell)$, the corresponding cumulative distribution functions (cdf) of the random variables $\Delta^{(n)}$ and $\Phi^{(n)}$, respectively. The goal of this article is to devise a numerical algorithm to write the two pmfs given in (23) and (24) or their corresponding cdfs. If the interest is on the two pmfs related to another information source- $n$ where $n \neq 1$, the same procedure can be repeated by renumbering the sources.

Our proposed methodology relies on constructing an infinite Markov chain of QBD type that produces cycles repeating forever in such a way that one cycle begins with the arrival of a source-1 packet that is chosen among the other arrivals to join either the server or the waiting room, and evolves until the reception of the second next successful class-1 packet. The level is always incremented until the second successful source1 packet reception instant at which we transition to an auxiliary state where the level is decremented until the level zero is hit in order to prepare for starting the next cycle. We will show that the steady-state distribution of this properly constructed QBD enables one to find the two pmfs given in (23) and (24) 
TABLE II

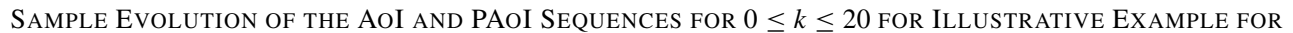
EACH OF THE THREE QUEUEING DISCIPLINES NPB, PB, AND NPSBR

\begin{tabular}{|c|c|c|c|c|c|c|}
\hline \multirow[b]{2}{*}{ Instant $k$} & \multicolumn{2}{|c|}{ NPB } & \multicolumn{2}{|c|}{ PB } & \multicolumn{2}{|c|}{ NPSBR } \\
\hline & $\Delta_{k}^{(1)}$ & $\Delta_{k}^{(2)}$ & $\Delta_{k}^{(1)}$ & $\Delta_{k}^{(2)}$ & $\Delta_{k}^{(1)}$ & $\Delta_{k}^{(2)}$ \\
\hline 0 & 0 & 0 & 0 & 0 & 0 & 0 \\
\hline : & : & : & : & : & : & : \\
\hline 5 & 5 & 5 & 5 & 5 & 5 & 5 \\
\hline 6 & $5\left(\Phi_{1}^{(1)} \leftarrow 6\right)$ & 6 & 6 & 6 & $5\left(\Phi_{1}^{(1)} \leftarrow 6\right)$ & 6 \\
\hline 7 & 6 & 7 & $2\left(\Phi_{1}^{(1)} \leftarrow 7\right)$ & 7 & 6 & 7 \\
\hline 8 & 7 & 8 & 3 & 8 & $2\left(\Phi_{2}^{(1)} \leftarrow 7\right)$ & 8 \\
\hline 9 & 8 & 9 & 4 & 9 & 3 & 9 \\
\hline 10 & 9 & 10 & 5 & 10 & 4 & 10 \\
\hline 11 & 10 & $4\left(\Phi_{1}^{(2)} \leftarrow 11\right)$ & 6 & 11 & 5 & 11 \\
\hline 12 & 11 & 5 & 7 & 12 & 6 & $4\left(\Phi_{1}^{(2)} \leftarrow 12\right)$ \\
\hline 13 & 12 & 6 & 8 & 13 & 7 & 5 \\
\hline 14 & 13 & 7 & 9 & 14 & 8 & 6 \\
\hline 15 & 14 & $2\left(\Phi_{2}^{(2)} \leftarrow 8\right)$ & 10 & $2\left(\Phi_{1}^{(2)} \leftarrow 15\right)$ & 9 & 7 \\
\hline 16 & 15 & 3 & 11 & 3 & 10 & 8 \\
\hline 17 & 16 & 4 & 12 & 4 & 11 & 9 \\
\hline 18 & $1\left(\Phi_{2}^{(1)} \leftarrow 17\right)$ & 5 & $1\left(\Phi_{2}^{(1)} \leftarrow 13\right)$ & 5 & 12 & 10 \\
\hline 19 & 2 & 6 & 2 & 6 & $10\left(\Phi_{3}^{(1)} \leftarrow 13\right)$ & 11 \\
\hline 20 & 3 & 7 & 3 & 7 & 11 & 12 \\
\hline$\vdots$ & $\vdots$ & $\vdots$ & & $\vdots$ & : & $\vdots$ \\
\hline
\end{tabular}

TABLE III

DESCRIPTION OF THE FIVE SUbSETS OF PHASES USED For NPB

\begin{tabular}{|l|l|l|}
\hline Subset & Phase & Description \\
\hline $\mathcal{S}_{1}$ & $(1, i)$ & $\begin{array}{l}\text { The first source-1 packet is in service and the service } \\
\text { phase is } i, 1 \leq i \leq r\end{array}$ \\
\hline $\mathcal{S}_{2}$ & 2 & $\begin{array}{l}\text { The service of first source-1 packet is over and the } \\
\text { server is idle }\end{array}$ \\
\hline $\mathcal{S}_{3}$ & $(3, i)$ & $\begin{array}{l}\text { The service of first source-1 packet is over, the } \\
\text { second source-1 packet is in service, and the service } \\
\text { phase is } i, 1 \leq i \leq r\end{array}$ \\
\hline $\mathcal{S}_{4}$ & $(4, i)$ & $\begin{array}{l}\text { The service of first source-1 packet is over, a source- } \\
n \text { packet }(n \neq 1) \text { is in service, and the service phase } \\
\text { is } i, 1 \leq i \leq r\end{array}$ \\
\hline $\mathcal{S}_{5}$ & 5 & $\begin{array}{l}\text { The service of the second source-1 packet is over } \\
\text { and we prepare for the next cycle }\end{array}$ \\
\hline
\end{tabular}

for each queueing discipline of interest. Recall that source$n$ information packet generation is governed by a Bernoulli process with parameter $p_{n}$ with $\bar{p}_{n}=1-p_{n}$. We also let $p=\sum_{n=1}^{N} p_{n}$. The service time $\Theta \sim \operatorname{DPH}(\boldsymbol{\alpha}, \boldsymbol{T}, \boldsymbol{t})$ of order $r$ with $E[\Theta]=\boldsymbol{\alpha}(\boldsymbol{I}-\boldsymbol{T})^{-1} \mathbf{1}=q^{-1}$. The overall load on the system, denoted by $\rho$, is defined as $\rho=p / q$.

\section{A. Nonpreemptive Bufferless Queueing System}

For NPB, we propose an infinite Markov chain $X_{k}=$ $\left(L_{k}, P_{k}\right), k=0,1, \ldots$ of QBD type characterized as in (7) with five subsets of phases. Table III describes each of the five subsets $\mathcal{S}_{v}, 1 \leq v \leq 5$, and the phases in each subset for the NPB system. For the construction of QBD, we define the pgf of the number of packet arrivals from all sources other than source-1

$$
\tau^{*}(z)=\prod_{n=2}^{N}\left(\bar{p}_{n}+p_{n} z\right)=\sum_{j=0}^{N-1} \tau_{j} z^{j} .
$$

Next, we define $\gamma_{0}=\prod_{n=1}^{N} \bar{p}_{n}$ to be the probability of no packet arrivals at a time instant and we also define

$$
\gamma_{1}=\sum_{j=0}^{N-1} p_{1} \frac{\tau_{j}}{j+1}
$$

to be the probability of a source-1 packet to be chosen (uniformly at random) among all packet arrivals. We also define $\gamma_{2}=1-\gamma_{0}-\gamma_{1}$, to be the probability of a source- $n$ packet $(n \neq 1)$ to be chosen among all packet arrivals. The proposed QBD evolves in the form of repetitive cycles each of which begins with the arrival of a source-1 packet into an empty system and continues until the reception of the next successful source-1 packet. At the beginning of a cycle, the level is zero and we are at phase $(1, i)$ for some $i$. A transition to phase $(1, j)$ occurs with probability $T(i, j)$, to phase 2 with probability $\gamma_{0} t(i)$, to phase $(3, j)$ with probability $\gamma_{1} t(i) \alpha(j)$, and to phase $(4, j)$ with probability $\gamma_{2} t(i) \alpha(j)$ while incrementing the level. While in phase 2 , we stay at phase 2 until an information packet arrival occurs. Therefore, in phase 2 , a transition to phase $(3, j)$ occurs with probability $\gamma_{1} \alpha(j)$, or to phase $(4, j)$ with probability $\gamma_{2} \alpha(j)$. The level is still incremented in all these transitions at phase 2 . At phase $(3, i)$, we transition to phase 5 with probability $t(i)$ when the service time of the second successful source-1 packet is over and to state $(3, j)$ with probability $T(i, j)$. The level is still incremented for all the phases of $\mathcal{S}_{3}$. While in phase $(4, i)$, a transition to phase $(4, j)$ occurs with probability $T(i, j)+\gamma_{2} t(i) \alpha(j)$, or to phase $(3, j)$ when the service completes and a source-1 packet arrival is chosen at the same instant that occurs with probability $\gamma_{1} t(i) \alpha(j)$, or to phase 2 when the service completes but there are no new packet arrivals that occurs with probability $\gamma_{0} t(i)$. Again, the level is incremented in all phases of $\mathcal{S}_{4}$. When in phase 5 , we stay at phase 5 while decrementing the 


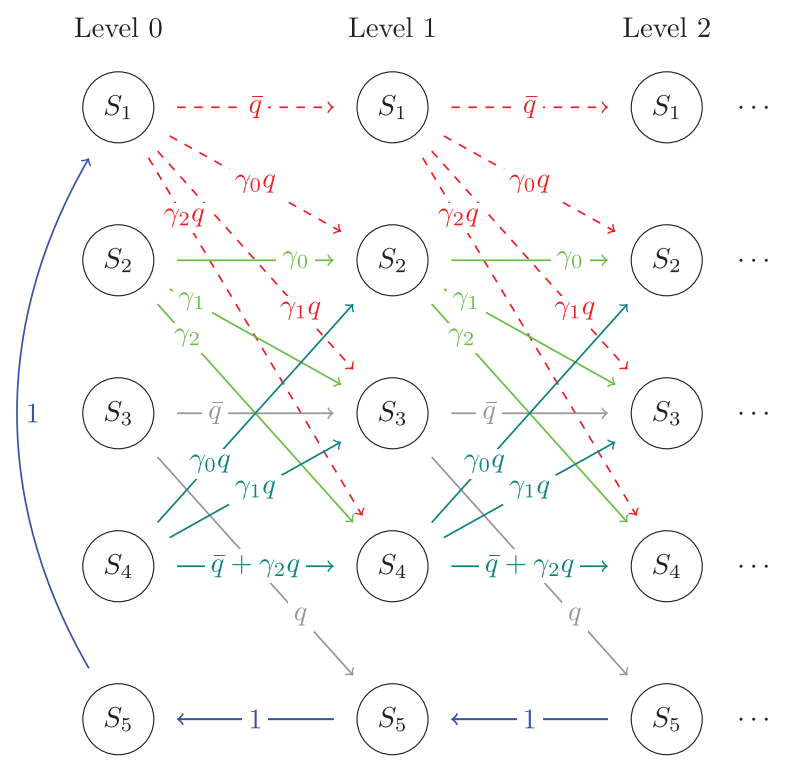

Fig. 2. DTMC transition diagram for the underlying QBD for the NPB queueing scheme when $\Theta \sim \operatorname{DPH}(1, q, \bar{q})$ for which the subset $\mathcal{S}_{i}$ consists of only one phase for each $i$.

level until the level zero is hit. When the level is zero, we make a transition to phase $(1, i)$ with probability $\alpha(i)$ while staying at level zero. This epoch is where a new cycle gets to begin. Fig. 2 presents the DTMC of QBD type for the case the service times are geometrically distributed with parameter $q$, i.e., $\Theta \sim \operatorname{DPH}(1, \bar{q}, q)$ with $\bar{q}=1-q$ for the underlying system where the arrows describe the transitions among the subsets (each of which consists of one single phase for the geometric service times) and across neighboring levels. With this description, the characterizing square matrices of size $(3 r+2)$ of the proposed QBD are as follows:

$$
\boldsymbol{A}_{\mathbf{0}}=\left[\begin{array}{ccccc}
\boldsymbol{T} & \gamma_{0} \boldsymbol{t} & \gamma_{1} \boldsymbol{t} \boldsymbol{\alpha} & \gamma_{2} \boldsymbol{t} \boldsymbol{\alpha} & \mathbf{0} \\
\mathbf{0} & \gamma_{0} & \gamma_{1} \boldsymbol{\alpha} & \gamma_{2} \boldsymbol{\alpha} & 0 \\
\mathbf{0} & \mathbf{0} & \boldsymbol{T} & \mathbf{0} & \boldsymbol{t} \\
\mathbf{0} & \gamma_{0} \boldsymbol{t} & \gamma_{1} \boldsymbol{t} \boldsymbol{\alpha} & \boldsymbol{T}+\gamma_{2} \boldsymbol{t} \boldsymbol{\alpha} & \mathbf{0} \\
\mathbf{0} & 0 & \mathbf{0} & \mathbf{0} & 0
\end{array}\right] .
$$

Moreover, $\boldsymbol{A}_{\mathbf{1}}$ is a matrix of zeros, $\boldsymbol{A}_{2}$ and $\boldsymbol{B}_{\mathbf{1}}$ are matrices of zeros except for the southeast block of size one which is one, and $\boldsymbol{B}_{\mathbf{0}}$ is a matrix of zeros except for its southwest block of size $1 \times r$, which is set to $\boldsymbol{\alpha}$. For the other two queueing systems, we will only provide the characterizing matrices for the underlying QBD. From the evolution of this QBD, we observe that the values that the level sequence $L_{k}$ of the proposed QBD takes in subsets $\mathcal{S}_{2}, \mathcal{S}_{3}$, and $\mathcal{S}_{4}$, in one QBDcycle, coincide with the sample values of the AoI process in its own cycles, as given in Table II. Recall that an AoI cycle starts with the age taking its initial value, which is the system time of a successful source-1 packet that is then subsequently incremented until the reception of the next successful source1 packet. The system time is composed of queue wait and service time of successful source-1 packets but in the case of NPB, the system time amounts to the service time of source1 packets. These observations lead us to the following main result for the NPB queueing system.
Theorem 1: Let $X_{k} \sim \operatorname{QBD}\left(\boldsymbol{B}_{\mathbf{0}}, \boldsymbol{B}_{\mathbf{1}}, \boldsymbol{A}_{\mathbf{0}}, \boldsymbol{A}_{\mathbf{1}}, \boldsymbol{A}_{\mathbf{2}}\right)$ having the characterizing matrices as in (26) with its stationary vector for level $k$ of size $1 \times(3 r+2)$ denoted by $\pi_{k}$ being in MG form $\pi_{\boldsymbol{k}}=\boldsymbol{\pi}_{\mathbf{0}} \boldsymbol{R}^{k} u_{k}$. Then, the steady-state pmf of the AoI sequence for the NPB system for source-1 is the same as that of $L^{\mathcal{S}}$, which is the steady-state level of the QBD process $X_{k}$ restricted to the subset $\mathcal{S}=\bigcup_{v=2}^{4} S_{v}$. Moreover, the steady-state pmf of the PAoI sequence for the NPB system for source-1 is the same as that of $1+L^{\mathcal{S}_{3}, 5}$ where $L^{\mathcal{S}_{3}, 5}$ is the steady-state level of the QBD process $X_{k}$ restricted to the pretransition epochs to state 5 from the subset $\mathcal{S}_{3}$.

Proof: The proof is based on sample path arguments. From the evolution of this QBD, we observe that the values that the level sequence $L_{k}$ of the proposed QBD takes in the phases belonging to the subsets $\mathcal{S}_{2}, \mathcal{S}_{3}$, and $\mathcal{S}_{4}$ in one QBDcycle, coincide with the sample values of the AoI process in its own cycles, as given in Table II. Similarly, one added to the values that the level sequence $L_{k}$ of the proposed QBD takes at the pretransition epochs from $\mathcal{S}_{3}$ to state 5, in one QBDcycle, coincide with the sample values of the PAoI sequence in one AoI cycle, as given in Table II. The pmfs of the AoI and PAoI sequences can then explicitly be written in MG form as in (15) and their factorial moments can explicitly be written as in (6).

\section{B. Preemptive Bufferless Queueing System}

For PB, we propose a similar infinite Markov chain $X_{k}=$ $\left(L_{k}, P_{k}\right), k=0,1, \ldots$ of QBD type characterized as in (7) with five subsets of phases, the description of the first four subsets being the same as that of NPB. On the other hand, for $\mathrm{PB}$, in phase 5 , either the service of the second source-1 packet is over and we prepare for the next cycle, or the first source-1 packet is unsuccessful, i.e., preempted during subset 1 , and after transitioning to phase 5, we prepare for the arrival of the first source-1 packet. This QBD evolves in the form of repetitive cycles each of which begins with the beginning of a source-1 packet's service and continues until the preemption of this packet or reception of the next successful source-1 packet. If the source-1 packet is unsuccessful during subset 1, i.e., preempted, then the cycle is called an unsuccessful cycle. Otherwise, the cycle continues until the reception of the second source-1 information packet and is called a successful cycle. At the beginning of a cycle, the level is zero and we are at phase 1 and the service of the source-1 packet is about to start. To describe the operation of the QBD, we first need the following definitions: $\gamma_{01}=\gamma_{0}+\gamma_{1}, \gamma_{02}=\gamma_{0}+\gamma_{2}, \gamma_{12}=$ $\gamma_{1}+\gamma_{2}$, and $\boldsymbol{\beta}=\boldsymbol{T} \mathbf{1}$. The differences from the NPB system due to preemption are now listed below in relation to the behavior at subsets of phases $\mathcal{S}_{1}, \mathcal{S}_{3}$, and $\mathcal{S}_{4}$.

1) At phase $(1, i)$, with probability $\gamma_{12} \beta(i)$, the first source1 packet gets to be preempted and we transition to phase 5 whereas the behavior at phase 5 is identical to that of the same phase in NPB. In this case, the cycle is an unsuccessful cycle.

2) At phase $(3, i)$, when the packet is preempted by a source- $n, n \neq 1$ packet, a transition to phase $(4, j)$ occurs due to preemption with probability $\gamma_{2} \beta(i) \alpha(j)$. This 
packet can be preempted by another source-1 packet in which case a transition to phase $(3, j)$ occurs due to preemption with probability $\gamma_{1} \beta(i) \alpha(j)$.

3) At phase $(4, i)$, when the packet is preempted by a source- $n, n \neq 1$ new packet, a transition to phase $(4, j)$ occurs due to preemption with probability $\gamma_{2} \beta(i) \alpha(j)$. When this packet is preempted by a source-1 packet, a transition to phase $(3, j)$ occurs due to preemption with probability $\gamma_{1} \beta(i) \alpha(j)$ and due to service completion with probability $\gamma_{1} t(i) \alpha(j)$. Additionally, we transition to phase 2 if the service completes and there are no new arrivals, which occurs with probability $\gamma_{0} t(i)$.

The characterizing matrix $\boldsymbol{A}_{\mathbf{0}}$ of the proposed QBD, $X_{k}$, is thus written as

$$
\boldsymbol{A}_{\mathbf{0}}=\left[\begin{array}{cccc}
\gamma_{0} \boldsymbol{T} & \gamma_{0} \boldsymbol{t} & \gamma_{1} \boldsymbol{t} \boldsymbol{\alpha} & \gamma_{2} \boldsymbol{t} \boldsymbol{\alpha} \\
\mathbf{0} & \gamma_{0} & \gamma_{1} \boldsymbol{\alpha} & \gamma_{2} \boldsymbol{\alpha} \\
\mathbf{0} & \mathbf{0} & \gamma_{0} \boldsymbol{T}+\gamma_{1} \boldsymbol{\beta} \boldsymbol{\alpha} & \gamma_{2} \boldsymbol{\beta} \boldsymbol{\alpha} \\
\mathbf{0} & \gamma_{0} \boldsymbol{t} & \gamma_{1} \mathbf{1} \boldsymbol{\alpha} & \gamma_{0} \boldsymbol{T}+\gamma_{2} \mathbf{1} \boldsymbol{\alpha} \\
\mathbf{0} & 0 & \mathbf{0} & \mathbf{0}
\end{array}\right.
$$

and the other characterizing matrices $\boldsymbol{A}_{\mathbf{1}}, \boldsymbol{A}_{\mathbf{2}}, \boldsymbol{B}_{\mathbf{1}}$, and $\boldsymbol{B}_{\mathbf{0}}$ being the same as in NPB. Thus, Theorem 1 stated for NPB also applies to PB with the exception that the $\boldsymbol{A}_{\mathbf{0}}$ matrix of the QBD for PB is given as in (27) as opposed to $\boldsymbol{A}_{\mathbf{0}}$ written as in (26) for NPB.

\section{Nonpreemptive Single Buffer With Replacement Queueing System}

Before introducing the proposed QBD for NPSBR as the other two queueing systems, we first need to obtain the behavior of the overall system upon the arrival of a source-1 packet. For this purpose, we introduce an auxiliary discrete-time random process $Y_{k} \in\{0,(1, i),(2, i), 1 \leq i \leq r\}$ for $k \geq 0$ where the first argument represents the number of information packets in the NPSBR system (in service and waiting room) at instant $k$ and the second argument stands for the phase of the service time unless the system is empty. It is not difficult to show that the random sequence $Y_{k}$ is governed by a DTMC with probability transition matrix $\boldsymbol{Q}$ of size $(2 r+1)$

$$
\boldsymbol{Q}=\left[\begin{array}{ccc}
\gamma_{0} & \gamma_{12} \boldsymbol{\alpha} & \mathbf{0} \\
\gamma_{0} \boldsymbol{t} & \gamma_{12} t \boldsymbol{\alpha}+\gamma_{0} \boldsymbol{T} & \gamma_{12} \boldsymbol{T} \\
0 & \boldsymbol{t} \boldsymbol{\alpha} & \boldsymbol{T}
\end{array}\right] .
$$

Let $\boldsymbol{x}=\left[\begin{array}{lll}x_{0} & \boldsymbol{y} & \boldsymbol{z}\end{array}\right]$ be the appropriately partitioned stationary vector of this DTMC satisfying

$$
\boldsymbol{x}=\boldsymbol{x} Q, \quad \boldsymbol{x} \mathbf{1}=1
$$

where $x_{0}$ is a scalar, and the row vectors $\boldsymbol{y}$ and $z$ are of size $r$. In the steady state, a chosen source-1 packet will find the system empty with probability $x_{0}$ and will immediately start to receive service. The same source-1 packet, upon arrival, will find the system at state $(1, i)$ with probability $x(i)$ and at state $(2, i)$ with probability $y(i)$.

For the NPSBR system, we propose an infinite Markov chain $X_{k}, k=0,1, \ldots$ of QBD type characterized as in (7) with ten subsets of phases denoted by $\mathcal{S}_{v}, 1 \leq v \leq 10$ for
TABLE IV

Description of THE TEN SUbSETS OF PHASEs Used For NPB

\begin{tabular}{|l|l|l|}
\hline Subset & Phase & Description \\
\hline $\mathcal{S}_{1}$ & $(1, i)$ & $\begin{array}{l}\text { A packet is in service and the service phase is } i, \\
1 \leq i \leq r, \text { and the first source-1 packet is in the } \\
\text { waiting room }\end{array}$ \\
\hline $\mathcal{S}_{2}$ & $(2, i)$ & $\begin{array}{l}\text { The first source-1 packet is in service and the service } \\
\text { phase is } i, 1 \leq i \leq r, \text { and the waiting room is empty }\end{array}$ \\
\hline $\mathcal{S}_{3}$ & $(3, i)$ & $\begin{array}{l}\text { The first source-1 packet is in service and the service } \\
\text { phase is } i, 1 \leq i \leq r, \text { and there is a source-1 packet } \\
\text { in the waiting room }\end{array}$ \\
\hline $\mathcal{S}_{4}$ & $(4, i)$ & $\begin{array}{l}\text { The first source-1 packet is in service and the service } \\
\text { phase is } i, 1 \leq i \leq r, \text { a source- } n \text { packet }(n \neq 1) \text { is } \\
\text { in the waiting room }\end{array}$ \\
\hline $\mathcal{S}_{5}$ & 5 & $\begin{array}{l}\text { The first source-1 packet service is over and we wait } \\
\text { for a packet arrival }\end{array}$ \\
\hline $\mathcal{S}_{6}$ & $(6, i)$ & $\begin{array}{l}\text { The second source-1 packet is in service and the } \\
\text { service phase is } i, 1 \leq i \leq r,\end{array}$ \\
\hline $\mathcal{S}_{7}$ & $(7, i)$ & $\begin{array}{l}\text { A source- } n \text { (n } \neq 1) \text { packet is in service and the } \\
\text { service phase is } i, 1 \leq i \leq r, \text { and the waiting room } \\
\text { is empty }\end{array}$ \\
\hline $\mathcal{S}_{8}$ & $(8, i)$ & $\begin{array}{l}\text { A source- } n \text { (n } \neq 1) \text { packet is in service and the } \\
\text { service phase is } i, 1 \leq i \leq r, \text { and there is a source- } \\
1 \text { packet in the waiting room }\end{array}$ \\
\hline $\mathcal{S}_{9}$ & $(9, i)$ & $\begin{array}{l}\text { A source- } n(n \neq 1) \text { packet is in service and the } \\
\text { service phase is } i, 1 \leq i \leq r, \text { and another source- } n \\
\text { packet }(n \neq 1) \text { is in the waiting room }\end{array}$ \\
\hline $\mathcal{S}_{10}$ & 10 & $\begin{array}{l}\text { The service of the second source-1 packet is over } \\
\text { and we prepare for the next cycle }\end{array}$ \\
\hline
\end{tabular}

which Table IV describes each of the ten subsets of phases used for the NPSBR system. This QBD evolves in the form of repetitive cycles as in the previous two queueing systems. A cycle begins with the arrival of a source-1 packet that immediately starts to receive service in $\mathcal{S}_{2}$ or is placed in the waiting room in subset $\mathcal{S}_{1}$ and continues until the reception of the second next successful source-1 packet. If the first source-1 packet is replaced by another packet while in the waiting room in a cycle, the cycle is said to be an unsuccessful cycle and a transition occurs to phase 10 for starting a new cycle. If the first source-1 packet starts receiving service, or equivalently a phase of $\mathcal{S}_{2}$ is once visited, then the cycle becomes a successful cycle. The characterizing matrix $\boldsymbol{A}_{\mathbf{0}}$ of size $(8 r+2)$ of the proposed QBD is given in (28), shown at the bottom of the next page. Moreover, $\boldsymbol{A}_{\mathbf{1}}$ is a matrix of zeros and $\boldsymbol{A}_{\mathbf{2}}$ and $\boldsymbol{B}_{\mathbf{1}}$ are matrices of zeros except for their southeast block entry being unity. On the other hand, $\boldsymbol{B}_{\mathbf{0}}$ is a matrix of zeros except for its southwest block, which is set to $(\boldsymbol{y}+\boldsymbol{z}) \boldsymbol{T}$ and its east-neighbor block, which is $(\boldsymbol{y}+\boldsymbol{z}) \boldsymbol{t} \boldsymbol{\alpha}+x_{0} \boldsymbol{\alpha}$. We now present the following main result for the NPSBR queueing system.

Theorem 2: Let $X_{k} \sim \operatorname{QBD}\left(\boldsymbol{B}_{\mathbf{0}}, \boldsymbol{B}_{\mathbf{1}}, \boldsymbol{A}_{\mathbf{0}}, \boldsymbol{A}_{\mathbf{1}}, \boldsymbol{A}_{\mathbf{2}}\right)$ having the characterizing matrices as in (28) with its stationary vector for level $k$ of size $1 \times(8 r+2)$ denoted by $\pi_{k}$ being in MG form $\pi_{k}=\pi_{0} R^{k} u_{k}$. Then, the steady-state pmf of the AoI sequence for the NPSBR system for source-1 is the same as that of $L^{\mathcal{S}}$, which is the steady-state level of the QBD process $X_{k}$ restricted to the subset $\mathcal{S}=\bigcup_{v=5}^{9} S_{v}$. Moreover, the steadystate pmf of the PAoI sequence for the NPSBR system for source-1 is the same as that of $1+L^{\mathcal{S}_{6}, 10}$ where $L^{\mathcal{S}_{6}, 10}$ is the steady-state level of the the QBD process $X_{k}$ restricted to the pretransition epochs to state 10 from the subset $\mathcal{S}_{6}$.

Proof: The proof is again based on sample path arguments. The system time of successful source-1 packets is 
composed of the time spent in the waiting room (in the phases of the subset $\mathcal{S}_{1}$ ) and during service (in the phases of the three subsets $\mathcal{S}_{2}, \mathcal{S}_{3}$, and $\mathcal{S}_{4}$ ) in successful cycles. In a successful cycle, the subset $\mathcal{S}_{2}$ needs to be visited. Subsequent to the visit of $\mathcal{S}_{2}$ in a successful cycle, the QBD evolves until the completion of the next successful source-1 packet's service completion (visiting subsets of phases $\mathcal{S}_{2}$ through $\mathcal{S}_{9}$ in successful cycles). Finally, in phase 10, we prepare for the next cycle. From the evolution of this QBD, we observe that the values that the level process of the proposed QBD takes in the subsets of phases $\mathcal{S}_{5}$ to $\mathcal{S}_{9}$ in one QBD cycle, coincide with the sample values of the AoI process in its own cycles, as given in Table II. Similarly, when we add one to the level sequence $L_{k}$ of the proposed QBD at only the pretransition epochs from $\mathcal{S}_{6}$ to state 10 , in one QBD-cycle, we also obtain the sample values of the PAoI sequence in one AoI cycle, as given in Table II. Transition to state 10 can only happen when the level is incremented. Therefore, the expressions (21) and (22) can be used to obtain the PAoI distribution of source-1 and subsequently its factorial moments can be computed from the expression (6).

\section{NUMERICAL RESUlts}

\section{A. Validation}

In this section, the analytical models we have proposed for the three queueing disciplines NPB, PB, and NPSBR are validated by simulations. In the first numerical example, we fix $N=3$ and we employ geometric service times with parameter $q=0.1$. Subsequently, for a given value of the load parameter $\rho$, we set the Bernoulli arrival vector of probabilities $\left(p_{1}, p_{2}, p_{3}\right)=((p / 7),(2 p / 7),(4 p / 7))$ where $p=q \rho$. The cdfs of the AoI and PAoI random sequences for the three different queueing schemes obtained with the proposed analytical model and simulations are depicted in Figs. 3 and 4, respectively, for $\rho=0.5$ and $\rho=2$, respectively. Furthermore, for the same numerical example, three service time distributions, namely, the deterministic and uniform distributions, along with the geometric distribution are employed with $E[\Theta]=q^{-1}=10$ and the load parameter $\rho$ is set to one. Among the three distributions, the deterministic distribution is the least variable, i.e., its coefficient of variation $(\mathrm{CoV})$, denoted by $c_{\Theta}$, defined as the ratio of its standard deviation to its mean, is zero, whereas the geometric distribution is the most variable among the three distributions. The corresponding cdfs for the AoI and PAoI proccess for source-1 only are depicted in Fig. 5.
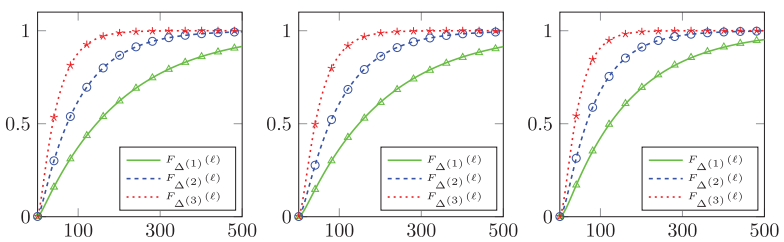

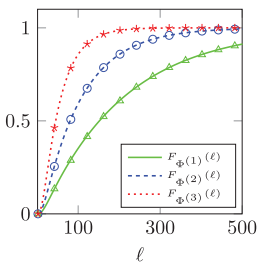

(a)

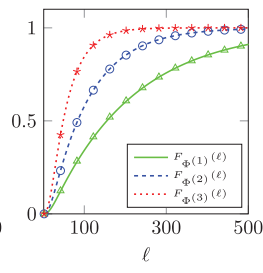

(b) (c)

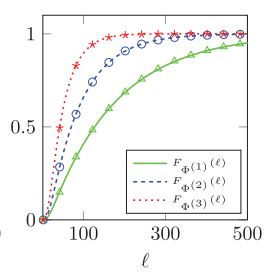

Fig. 3. Cdfs of AoI and PAoI processes for the three queueing schemes obtained using the analytical model and simulations (denoted by markers) when $\rho=0.5$. (a) PB. (b) NPB. (c) NPSBR.
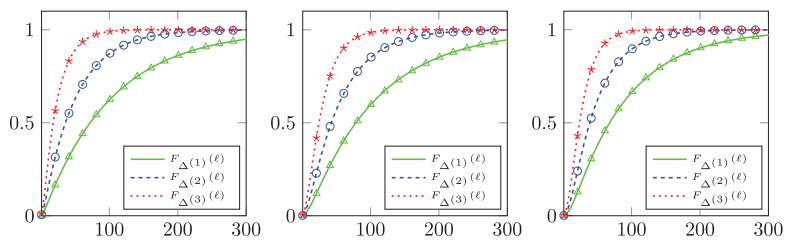

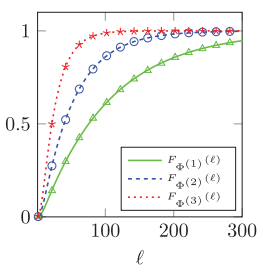

(a)

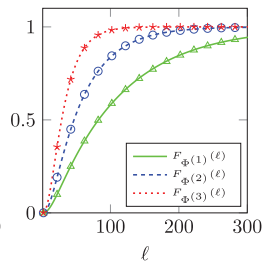

(b)

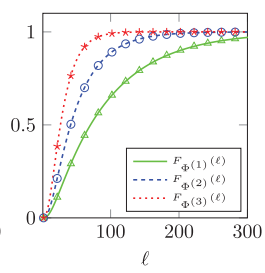

(c)
Fig. 4. Cdfs of AoI and PAoI processes for the three queueing schemes obtained using the analytical model and simulations (denoted by markers) when $\rho=2$. (a) PB. (b) NPB. (c) NPSBR.

For all the validation examples, we have observed a perfect match between the results obtained with the proposed analytical model and the simulation results. We also observe that the PB queue management scheme is outperformed by the NPB system when service times have relatively lower variability but this observation is reversed when the related variability increases, i.e., when service times are geometrically distributed. Moreover, the single buffer queueing discipline is shown to perform best in the investigated scenarios.

$$
\boldsymbol{A}_{\mathbf{0}}=\left[\begin{array}{cccccccccc}
\gamma_{0} \boldsymbol{T} & \gamma_{0} t \boldsymbol{\alpha} & \mathbf{0} & \mathbf{0} & \mathbf{0} & \mathbf{0} & \mathbf{0} & \mathbf{0} & \mathbf{0} & \gamma_{12} \mathbf{1} \\
\mathbf{0} & \gamma_{0} \boldsymbol{T} & \gamma_{1} \boldsymbol{T} & \gamma_{2} \boldsymbol{T} & \gamma_{0} t & \gamma_{1} t \boldsymbol{\alpha} & \gamma_{2} t \boldsymbol{\alpha} & \mathbf{0} & \mathbf{0} & \mathbf{0} \\
\mathbf{0} & \mathbf{0} & \gamma_{01} \boldsymbol{T} & \gamma_{2} \boldsymbol{T} & \mathbf{0} & \gamma_{01} t \boldsymbol{\alpha} & \gamma_{2} t \boldsymbol{\alpha} & \mathbf{0} & \mathbf{0} & \mathbf{0} \\
\mathbf{0} & \mathbf{0} & \gamma_{1} \boldsymbol{T} & \gamma_{02} \boldsymbol{T} & \mathbf{0} & \gamma_{1} t \boldsymbol{\alpha} & \gamma_{02} t \boldsymbol{\alpha} & \mathbf{0} & \mathbf{0} & \mathbf{0} \\
\mathbf{0} & \mathbf{0} & \mathbf{0} & \mathbf{0} & \gamma_{0} & \gamma_{1} \boldsymbol{\alpha} & \gamma_{2} \boldsymbol{\alpha} & \mathbf{0} & \mathbf{0} & 0 \\
\mathbf{0} & \mathbf{0} & \mathbf{0} & \mathbf{0} & \mathbf{0} & \boldsymbol{T} & \mathbf{0} & \mathbf{0} & \mathbf{0} & \boldsymbol{t} \\
\mathbf{0} & \mathbf{0} & \mathbf{0} & \mathbf{0} & \gamma_{0} t & \gamma_{1} t \boldsymbol{\alpha} & \gamma_{0} \boldsymbol{T}+\gamma_{2} t \boldsymbol{\alpha} & \gamma_{1} \boldsymbol{T} & \gamma_{2} \boldsymbol{T} & \mathbf{0} \\
\mathbf{0} & \mathbf{0} & \mathbf{0} & \mathbf{0} & \mathbf{0} & \gamma_{01} t \boldsymbol{\alpha} & \gamma_{2} t \boldsymbol{\alpha} & \gamma_{01} \boldsymbol{T} & \gamma_{2} \boldsymbol{T} & \mathbf{0} \\
\mathbf{0} & \mathbf{0} & \mathbf{0} & \mathbf{0} & \mathbf{0} & \gamma_{1} t \boldsymbol{\alpha} & \gamma_{02} t \boldsymbol{\alpha} & \gamma_{1} \boldsymbol{T} & \gamma_{02} \boldsymbol{T} & \mathbf{0} \\
\mathbf{0} & \mathbf{0} & \mathbf{0} & \mathbf{0} & \mathbf{0} & \mathbf{0} & \mathbf{0} & \mathbf{0} & \mathbf{0} & 0
\end{array}\right]
$$



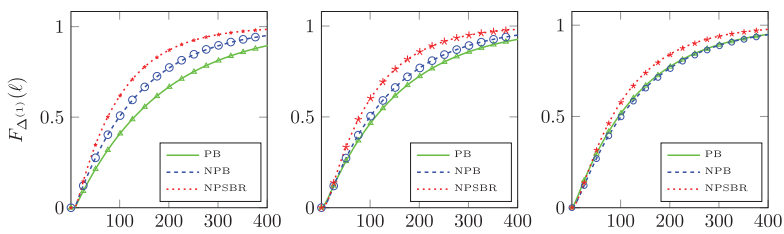

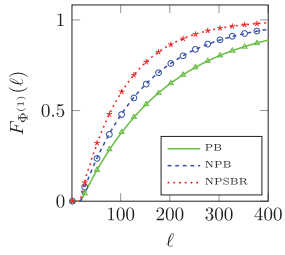

(a)

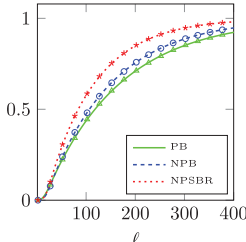

(b)

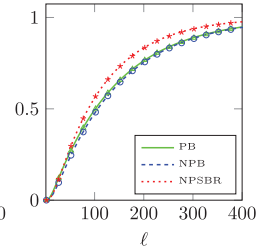

(c)
Fig. 5. Cdfs of source-1 AoI and PAoI processes for the three queueing systems obtained by the analytical model and simulations (denoted by markers) for the three service time distributions when $\rho=1$ : (a) deterministic, (b) uniform, and (c) geometric service times.

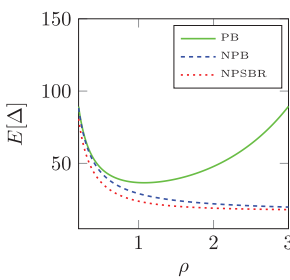

(a)

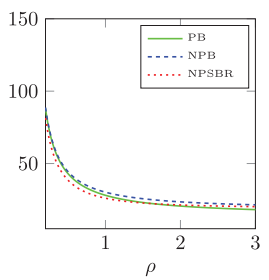

(b)

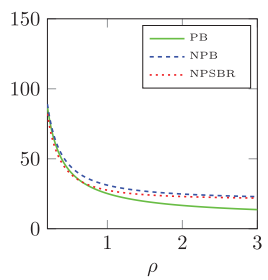

(c)
Fig. 6. Mean AoI $E[\Delta]$ across the three sources as function of the system load $\rho$ : (a) deterministic, (b) geometric, (c) mixed geometric service times, when $q=0.2, p=q \rho$, and $\left(p_{1}, p_{2}, p_{3}\right)$ is set to $((p / 3),(p / 3),(p / 3))$.

\section{B. Numerical Examples}

In this section, we use the solution of the analytical models to gain insight into the operation of the three queueing schemes and simulation results are not reported. In the first numerical example, we depict, in Figs. 6 and 7, the average AoI across all sources, namely $E[\Delta]=(1 / N) \sum_{n=1}^{N} E\left[\Delta^{(n)}\right]$, for a system with $N=3$ sources, as a function of the system load $\rho$ for the three queueing disciplines. Three service time distributions are used for each case with $E[\Theta]=q^{-1}=5$, namely, the deterministic distribution with $c_{\Theta}=0$, geometric distribution with $c_{\Theta}=0.89$, and the mixed geometric distribution for which the service time is either geometric with parameter 2 or with parameter 8 , each with probability 0.5 , giving rise to $c_{\Theta}=1.23$. The Bernouilli arrival vector $\left(p_{1}, p_{2}, p_{3}\right)$ is set to $((p / 3),(p / 3),(p / 3))$ and $((p / 7),(2 p / 7),(4 p / 7))$ in Figs. 6 and 7 , respectively. For the PB system, $E[\Delta]$ first drops with increased $\rho$ but beyond a certain value of $\rho$, the mean AoI starts to rise again in the deterministic service time case. In the geometric distribution case, the performance of the PB system improves substantially outperforming the NPB system but it is still outperformed by the NPSBR system except for very high loads. However, in the mixed geometric case with a higher $\mathrm{CoV}$ value, the $\mathrm{PB}$ discipline outperforms the other two queueing disciplines for all values of $\rho$ above a moderate threshold. At low loads, keeping packets in the waiting room as in NPSBR appears to be very important to keep the system busy. Therefore, low load regimes favor NPSBR. However, at higher loads and for higher $\mathrm{CoV}$ values of the service time, the PB system starts to take over. These observations are valid for

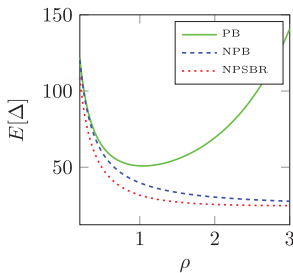

(a)

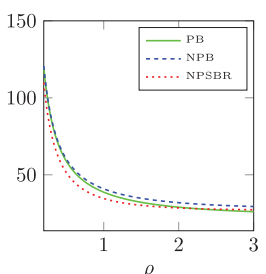

(b)

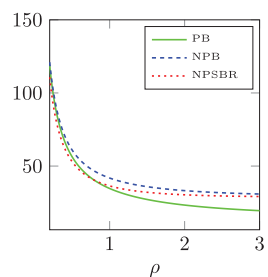

(c)
Fig. 7. Mean AoI $E[\Delta]$ across the three sources as function of the system load $\rho$ : (a) deterministic, (b) geometric, (c) mixed geometric service times, when $q=0.2, p=q \rho$, and $\left(p_{1}, p_{2}, p_{3}\right)$ is set to $((p / 7),(2 p / 7),(4 p / 7))$.

both arrival vectors in Figs. 6 and 7, corresponding to different traffic mixes. However, when the traffic mixture is not even across sources, the mean AoI increases since the AoI of low intensity sources increases substantially in these cases leading to increased mean AoI figures.

In all the previous examples, the arrival probabilities were fixed. In the final numerical example, we study the impact of the choice of the per-source packet generation probabilities $p_{1}$ and $p_{2}$ on the system cost $C(\alpha)$ for a two-source system with geometrically distributed service times only, which is given in the following form:

$$
C(\alpha)=E\left[\Delta^{(1)}\right]+\alpha E\left[\Delta^{(2)}\right], 0 \leq \alpha \leq 1
$$

which allows one to give more importance to source-1 over source- 2 with a proper choice of the cost parameter $\alpha$. When $\alpha=1$, both sources are equally important whereas when $\alpha \rightarrow 0$, the mean age of the second source becomes less relevant. For a given cost parameter $\alpha$, we do exhaustive search to find the optimum packet generation probabilities $p_{1}^{*}$ and $p_{2}^{*}$ that minimize the system cost $C(\alpha)$ where the minimum attainable cost is denoted by $C^{*}(\alpha)$. Table $\mathrm{V}$ tabulates the optimum values $p_{1}^{*}, p_{2}^{*}$, and $C^{*}(\alpha)$ for various values of $\alpha$, for three different values of the service time parameter $q$, and for each of the three queueing disciplines of interest. As a second scenario, for power budgeting, we impose a bound on the overall packet generation rate while doing exhaustive search, i.e., $p=p_{1}+p_{2} \leq \beta$, where $\beta$ is power constraint parameter since packet generation requires a certain energy consumption. In Table VI, we provide our results for the specific case of $\beta=0.1$. Studying Tables V and VI, we have the following observations.

1) The optimum values of $p_{1}$ and $p_{2}$ turn out to be the same for all the three queueing disciplines but the optimum cost values are not necessarily the same.

2) In the absence of a power constraint, the optimum packet generation rate for source-1 is always one and that of source- 2 is one only when $\alpha=1$ and it decreases when $\alpha$ decreases. Since $p_{1}^{*}=1$ in all these cases, there is always a packet arrival at each time instant and the two queueing disciplines NPB and NPSBR behave exactly the same under the same set of traffic parameters. In this case, the PB always outperforms the other two disciplines when the optimum packet generation rates are employed, thanks to the assumption of geometric service times. 
TABLE V

OPTIMUM Values of $p_{1}$ AND $p_{2}$ AND THE Optimum Value of $C(\alpha)$ FOR THREE DIFFERENT VALUES OF $q \in\{0.05,0.1,0.25\}$ FOR VARIOUS VALUES OF $\alpha$ FOR THE THREE QUEUEING DISCIPLINES IN THE AbSENCE of POWER CONSTRAint

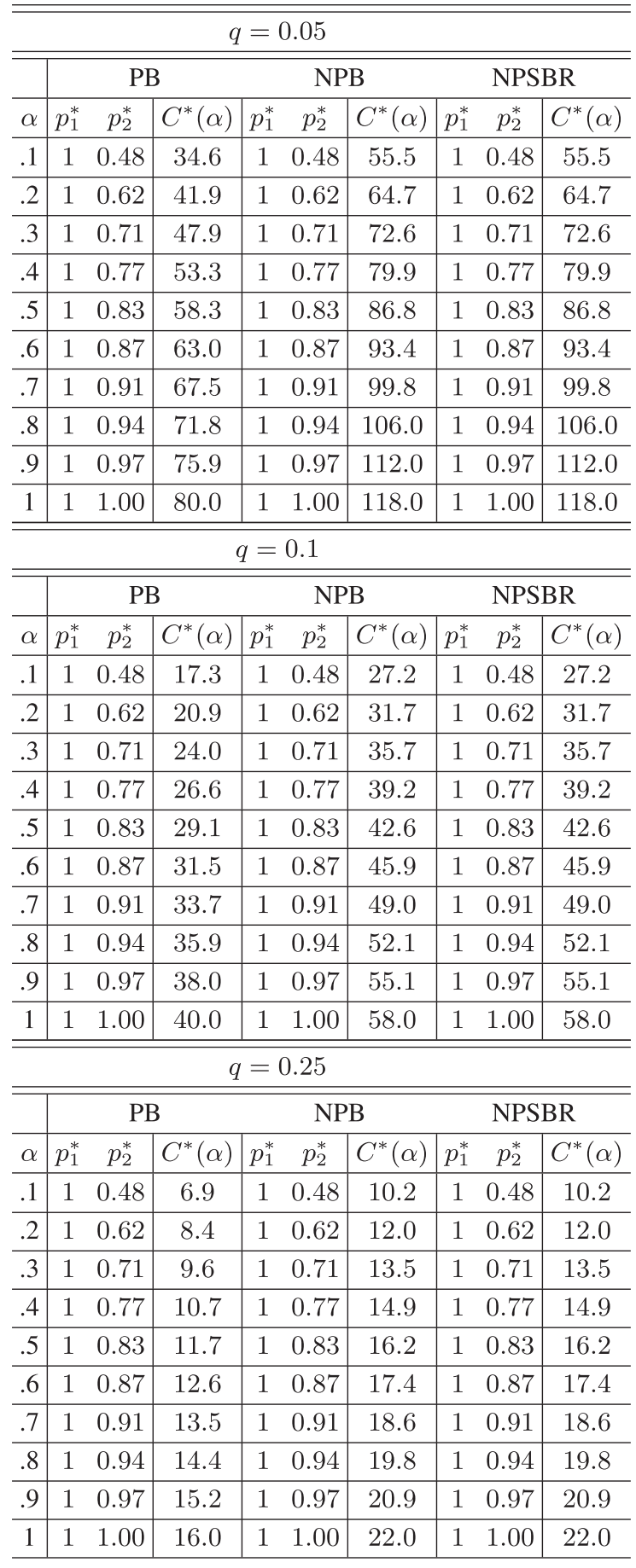

3) When there is a power constraint imposed on the packet generation rates, i.e., $\beta=0.1$, the situation is very different. In this case, NPSBR always outperforms NPB and there are situations when NPSBR outperforms PB that appear to occur for lower average service times.
TABLE VI

OPtimum Values of $p_{1}$ AND $p_{2}$ AND The OPtimum VALUE OF $C(\alpha)$ FOR THREE DIFFERENT VALUES OF $q \in\{0.05,0.1,0.25\}$ FOR VARIOUS VALUES OF $\alpha$ FOR THE THREE QUEUEING Disciplines. THE POWER CONSTRAINT IS TAKEN AS $p_{1}+p_{2} \leq \beta=0.1$

\begin{tabular}{c|cc|c|cc|c|cc|c}
\hline \hline \multicolumn{10}{c}{$q=0.05$} \\
\hline \hline & \multicolumn{1}{c}{ PB } \\
\hline$\alpha$ & $\begin{array}{c}p_{1}^{*} \\
\left(\times 10^{-2}\right)\end{array}$ & $p_{2}^{*}$ & $C^{*}(\alpha)$ & $\begin{array}{l}p_{1}^{*} \\
\left(\times 10^{-2}\right)\end{array}$ & $p_{2}^{*}$ & $C^{*}(\alpha)$ & $p_{1}^{*}$ & $p_{2}^{*}$ \\
$\left(\times 10^{-2}\right)$ & $C^{*}(\alpha)$ \\
\hline .1 & 7.6 & 2.4 & 50.6 & 7.6 & 2.4 & 64.9 & 7.6 & 2.4 & 61.7 \\
\hline .2 & 6.9 & 3.1 & 61.2 & 6.9 & 3.1 & 76.8 & 6.9 & 3.1 & 72.0 \\
\hline .3 & 6.4 & 3.6 & 70.0 & 6.4 & 3.6 & 86.9 & 6.4 & 3.6 & 81.0 \\
\hline .4 & 6.1 & 3.9 & 77.9 & 6.1 & 3.9 & 96.1 & 6.1 & 3.9 & 89.2 \\
\hline .5 & 5.8 & 4.2 & 85.2 & 5.8 & 4.2 & 104.7 & 5.8 & 4.2 & 97.0 \\
\hline .6 & 5.6 & 4.4 & 92.1 & 5.6 & 4.4 & 112.9 & 5.6 & 4.4 & 104.4 \\
\hline .7 & 5.4 & 4.6 & 98.7 & 5.4 & 4.6 & 120.8 & 5.4 & 4.6 & 111.5 \\
\hline .8 & 5.3 & 4.7 & 105.0 & 5.3 & 4.7 & 128.4 & 5.3 & 4.7 & 118.5 \\
\hline .9 & 5.1 & 4.9 & 111.1 & 5.1 & 4.9 & 135.8 & 5.1 & 4.9 & 125.3 \\
\hline 1 & 5.0 & 5.0 & 117.0 & 5.0 & 5.0 & 143.0 & 5.0 & 5.0 & 131.9 \\
\hline \hline \multicolumn{10}{|c|}{$q=0.1$} \\
\hline \hline
\end{tabular}

\begin{tabular}{c|cc|c|cc|c|cc|c}
\hline \hline & \multicolumn{4}{|c}{ PB } & \multicolumn{4}{c}{ NPB } & \multicolumn{3}{c}{ NPSBR } \\
\hline$\alpha$ & $\begin{array}{l}p_{1}^{*} \\
\left(\times 10^{-2}\right)\end{array}$ & $C^{*}(\alpha)$ & $\begin{array}{l}p_{1}^{*} \\
\left(\times 10^{-2}\right)\end{array}$ & $C^{*}(\alpha)$ & $\begin{array}{l}p_{1}^{*} \\
\left(\times 10^{-2}\right)\end{array}$ & $C^{*}(\alpha)$ \\
\hline .1 & 7.6 & 2.4 & 33.2 & 7.6 & 2.4 & 38.4 & 7.6 & 2.4 & 34.8 \\
\hline .2 & 6.9 & 3.1 & 40.3 & 6.9 & 3.1 & 45.9 & 6.9 & 3.1 & 41.0 \\
\hline .3 & 6.4 & 3.6 & 46.1 & 6.4 & 3.6 & 52.2 & 6.4 & 3.6 & 46.3 \\
\hline .4 & 6.1 & 3.9 & 51.3 & 6.1 & 3.9 & 57.8 & 6.1 & 3.9 & 51.2 \\
\hline .5 & 5.8 & 4.2 & 56.1 & 5.8 & 4.2 & 63.1 & 5.8 & 4.2 & 55.7 \\
\hline .6 & 5.6 & 4.4 & 60.6 & 5.6 & 4.4 & 68.1 & 5.6 & 4.4 & 60.1 \\
\hline .7 & 5.4 & 4.6 & 65.0 & 5.4 & 4.6 & 72.9 & 5.4 & 4.6 & 64.2 \\
\hline .8 & 5.3 & 4.7 & 69.1 & 5.3 & 4.7 & 77.5 & 5.3 & 4.7 & 68.3 \\
\hline .9 & 5.1 & 4.9 & 73.1 & 5.1 & 4.9 & 82.0 & 5.1 & 4.9 & 72.2 \\
\hline 1 & 5.0 & 5.0 & 77.0 & 5.0 & 5.0 & 86.4 & 5.0 & 5.0 & 76.0 \\
\hline \hline
\end{tabular}

\begin{tabular}{c|cc|c|cc|c|cc|c}
\hline \hline & \multicolumn{4}{|c}{ PB NPB } & \multicolumn{4}{c}{ NPSBR } \\
\hline$\alpha$ & $\begin{array}{l}p_{1}^{*} \\
\left(\times 10^{-2}\right)\end{array}$ & $p^{*}(\alpha)$ & $\begin{array}{l}p_{1}^{*} \\
\left(\times 10^{-2}\right)\end{array}$ & $C^{*}(\alpha)$ & $\begin{array}{l}p_{1}^{*} \\
\left(\times 10^{-2}\right)\end{array}$ & $C^{*}(\alpha)$ \\
\hline .1 & 7.6 & 2.4 & 22.8 & 7.6 & 2.4 & 23.8 & 7.6 & 2.4 & 21.9 \\
\hline .2 & 6.9 & 3.1 & 27.7 & 6.9 & 3.1 & 28.8 & 6.9 & 3.1 & 26.3 \\
\hline .3 & 6.5 & 3.5 & 31.7 & 6.5 & 3.5 & 32.9 & 6.5 & 3.5 & 29.9 \\
\hline .4 & 6.1 & 3.9 & 35.3 & 6.1 & 3.9 & 36.6 & 6.1 & 3.9 & 33.2 \\
\hline .5 & 5.9 & 4.1 & 38.6 & 5.9 & 4.1 & 40.0 & 5.9 & 4.1 & 36.2 \\
\hline .6 & 5.6 & 4.4 & 41.7 & 5.6 & 4.4 & 43.2 & 5.6 & 4.4 & 39.1 \\
\hline .7 & 5.4 & 4.6 & 44.7 & 5.4 & 4.6 & 46.3 & 5.4 & 4.6 & 41.8 \\
\hline .8 & 5.3 & 4.7 & 47.6 & 5.3 & 4.7 & 49.2 & 5.3 & 4.7 & 44.5 \\
\hline .9 & 5.1 & 4.9 & 50.3 & 5.1 & 4.9 & 52.1 & 5.1 & 4.9 & 47.1 \\
\hline 1 & 5.0 & 5.0 & 53.0 & 5.0 & 5.0 & 54.8 & 5.0 & 5.0 & 49.6 \\
\hline
\end{tabular}

\section{CONCLUSION}

In this article, we proposed a discrete-time queueing model to derive the exact distributions of the AoI and PAoI sequences in a multisource IoT-based status update system with Bernoulli 
information packet arrivals and DPH service times. Three queueing disciplines are considered, namely, NPB, PB, and NPSBR. Numerical examples along with simulations are presented to validate the proposed approach. We showed that the preemptive PB scheme does not work as well for low loads and for relatively less variable service times such as the deterministic or uniform service time distributions but it becomes more effective when the $\mathrm{CoV}$ of the service time increases. On the other hand, the NPSBR scheme outperforms the other queueing disciplines for the case of low loads and service time distributions with lower CoV. Using the proposed analytical model, we also provided a numerical example (using geometric service times only) on the optimum choice of the Bernoulli parameters in a practical IoT system with two sources with diverse AoI requirements and the three queueing disciplines are compared and contrasted in this setting. We have shown that the PB system yields the best performance in majority of the cases whereas it is slightly outperformed by the NPSBR discipline in scenarios with power constraints and relatively shorter average service times.

\section{REFERENCES}

[1] S. K. Kaul, M. Gruteser, V. Rai, and J. Kenney, "Minimizing age of information in vehicular networks," in Proc. 8th Annu. IEEE Commun. Soc. Conf. Sensor Mesh Ad Hoc Commun. Netw., Jun. 2011, pp. $350-358$.

[2] S. K. Kaul, R. D. Yates, and M. Gruteser, "Real-time status: How often should one update?" in Proc. IEEE INFOCOM, Mar. 2012, pp. $2731-2735$.

[3] S. K. Kaul, R. D. Yates, and M. Gruteser, "Status updates through queues," in Proc. 46th Annu. Conf. Inf. Sci. Syst. (CISS), Mar. 2012, pp. 1-6.

[4] N. Pappas, J. Gunnarsson, L. Kratz, M. Kountouris, and V. Angelakis, "Age of information of multiple sources with queue management," in Proc. IEEE Int. Conf. Commun. (ICC), Jun. 2015, pp. 5935-5940.

[5] A. Kosta, N. Pappas, A. Ephremides, and V. Angelakis, "Age and value of information: Non-linear age case," in Proc. IEEE Int. Symp. Inf. Theory (ISIT), Jun. 2017, pp. 326-330.

[6] M. Costa, M. Codreanu, and A. Ephremides, "On the age of information in status update systems with packet management," IEEE Trans. Inf. Theory, vol. 62, no. 4, pp. 1897-1910, Apr. 2016.

[7] K. Chen and L. Huang, "Age-of-information in the presence of error," in Proc. IEEE Int. Symp. Inf. Theory (ISIT), Jul. 2016, pp. 2579-2583.

[8] R. D. Yates and S. K. Kaul, "The age of information: Real-time status updating by multiple sources," IEEE Trans. Inf. Theory, vol. 65 , no. 3 , pp. 1807-1827, Mar. 2019.

[9] Y. Inoue, H. Masuyama, T. Takine, and T. Tanaka, "A general formula for the stationary distribution of the age of information and its application to single-server queues," IEEE Trans. Inf. Theory, vol. 65, no. 12, pp. 8305-8324, Dec. 2019.

[10] Y. Sun, E. Uysal-Biyikoglu, R. D. Yates, C. E. Koksal, and N. B. Shroff, "Update or wait: How to keep your data fresh," IEEE Trans. Inf. Theory, vol. 63, no. 11, pp. 7492-7508, Nov. 2017.

[11] L. Huang and E. Modiano, "Optimizing age-of-information in a multiclass queueing system," in Proc. IEEE Int. Symp. Inf. Theory (ISIT), Jun. 2015, pp. 1681-1685.

[12] A. Arafa and S. Ulukus, "Age minimization in energy harvesting communications: Energy-controlled delays," in Proc. 51st Asilomar Conf. Signals Syst. Comput., Oct. 2017, pp. 1801-1805.

[13] Y. Hsu, E. Modiano, and L. Duan, "Age of information: Design and analysis of optimal scheduling algorithms," in Proc. IEEE Int. Symp. Inf. Theory (ISIT), Jun. 2017, pp. 561-565.

[14] Q. He, D. Yuan, and A. Ephremides, "Optimal link scheduling for age minimization in wireless systems," IEEE Trans. Inf. Theory, vol. 64, no. 7, pp. 5381-5394, Jul. 2018.

[15] M. Costa, M. Codreanu, and A. Ephremides, "Age of information with packet management," in Proc. IEEE Int. Symp. Inf. Theory, 2014, pp. $1583-1587$

[16] P. Thubert, "An architecture for IPv6 over the TSCH mode of IEEE 802.15.4," Internet Eng. Task Force, Internet-Draft draft-ietf-6tisch-architecture-29, Aug. 2020. [Online]. Available: https://datatracker.ietf.org/doc/html/draft-ietf-6tisch-architecture-29
[17] D. Zorbas, K. Abdelfadeel, P. Kotzanikolaou, and D. Pesch, "TS-LoRa: Time-slotted LoRaWAN for the industrial Internet of Things," Comput. Commun., vol. 153, pp. 1-10, Mar. 2020.

[18] V. Tripathi, R. Talak, and E. Modiano, "Age of information for discrete time queues," 2019. [Online]. Available: arXiv:1901.10463.

[19] A. Bobbio, A. Horváth, M. Scarpa, and M. Telek, "Acyclic discrete phase type distributions: properties and a parameter estimation algorithm," Perform. Eval., vol. 54, no. 1, pp. 1-32, 2003.

[20] A. Kosta, N. Pappas, and V. Angelakis, "Age of information: A new concept, metric, and tool," Found. Trends ${ }^{\circledR}$ Netw., vol. 12, no. 3, pp. 162-259, 2017

[21] R. D. Yates and S. K. Kaul, "Real-time status updating: Multiple sources," in Proc. IEEE Int. Symp. Inf. Theory, Jul. 2012, pp. 2666-2670.

[22] N. Akar, O. Dogan, and E. U. Atay, "Finding the exact distribution of (peak) age of information for queues of $\mathrm{PH} / \mathrm{PH} / 1 / 1$ and $\mathrm{M} / \mathrm{PH} / 1 / 2$ type," IEEE Trans. Commun., vol. 68, no. 9, pp. 5661-5672, Sep. 2020.

[23] E. Najm and E. Telatar, "Status updates in a multi-stream M/G/1/1 preemptive queue," in Proc. IEEE INFOCOM IEEE Conf. Comput. Commun. Workshops (INFOCOM WKSHPS), 2018, pp. 124-129.

[24] S. Farazi, A. G. Klein, and D. R. Brown, "Average age of information in multi-source self-preemptive status update systems with packet delivery errors," in Proc. 53rd Asilomar Conf. Signals Syst. Comput., 2019, pp. 396-400.

[25] M. Moltafet, M. Leinonen, and M. Codreanu, "Average age of information for a multi-source $\mathrm{M} / \mathrm{M} / 1$ queueing model with packet management," 2020. [Online]. Available: arXiv:2001.03959.

[26] S. K. Kaul and R. D. Yates, "Timely updates by multiple sources: The M/M/1 queue revisited," in Proc. 54th Annu. Conf. Inf. Sci. Syst. (CISS), 2020, pp. 1-6.

[27] R. D. Yates, J. Zhong, and W. Zhang, "Updates with multiple service classes," in Proc. IEEE Int. Symp. Inf. Theory (ISIT), 2019, pp. 1017-1021.

[28] A. Kosta, N. Pappas, A. Ephremides, and V. Angelakis, "Queue management for age sensitive status updates," in Proc. IEEE Int. Symp. Inf. Theory (ISIT), 2019, pp. 330-334.

[29] A. Kosta, N. Pappas, A. Ephremides, and V. Angelakis, "Non-linear age of information in a discrete time queue: Stationary distribution and average performance analysis," 2020. [Online]. Available: arXiv:2002.08798.

[30] S. Greeuw, "On the relation between matrix-geometric and discrete phase-type distributions," M.S. thesis, Dept. Mathematics, Univ. Amsterdam, Amsterdam, The Netherlands, 2009.

[31] N. Akar, "Fitting matrix geometric distributions by model reduction," Stochastic Models, vol. 31, no. 2, pp. 292-315, 2015.

[32] M. F. Neuts, Matrix-Geometric Solutions in Stochastic Models. Baltimore, MD, USA: Johns Hopkins Univ. Press, 1981.

[33] G. Latouche and V. Ramaswami, "A logarithmic reduction algorithm for quasi-birth-death processes," J. Appl. Probab., vol. 30, no. 3, pp. 650-674, 1993.

[34] N. Akar, N. C. Oguz, and K. Sohraby, "A novel computational method for solving finite QBD processes," Commun. Stat. Stochastic Models, vol. 16, no. 2, pp. 273-311, 2000.

Nail Akar (Member, IEEE) received the B.S. degree in electrical and electronics engineering from Middle East Technical University, Ankara, Turkey, in 1987, and the M.S. and Ph.D. degrees in electrical and electronics engineering from Bilkent University, Ankara, in 1989 and 1994, respectively.

From 1994 to 1996, he was a Visiting Scholar and a Visiting Assistant Professor with the Computer Science Telecommunications Program, University of Missouri-Kansas City, Kansas City, MO, USA. He joined the Technology Planning and Integration group of Long Distance Division, Sprint, Overland Park, KS, USA, in 1996, where he held a Senior Member of technical staff position from 1999 to 2000. Since 2000, he has been with Bilkent University currently as a Professor of the Electrical and Electronics Engineering Department and as the Associate Dean of the Engineering Faculty. He visited the School of Computing, University of Missouri-Kansas City, as a Fulbright Scholar in 2010 for a period of six months. His research interests are on performance modeling of computer and communication systems and networks, wireless networks, Internet of Things, queueing theory, and optimization.

Ozancan Dogan received the B.S. degree in electrical and electronics engineering from Bilkent University, Ankara, Turkey, in 2018, where he is currently pursuing the M.S. degree.

His current research interest is on performance modeling of age of information in communication systems. 